\title{
Potential and cost of carbon sequestration in Indian agriculture: Estimates from long-term field experiments
}

\author{
H. Pathak*, K. Byjesh, B. Chakrabarti, P.K. Aggarwal \\ Division of Environmental Sciences, Indian Agricultural Research Institute, Pusa, New Delhi 110012, India
}

\section{A R T I C L E I N F O}

\section{Article history:}

Received 4 September 2010

Accepted 13 September 2010

\section{Keywords:}

Carbon sequestration

Benefit:cost analysis

Indian agriculture

Long-term experiments

Soil organic carbon

\begin{abstract}
A B S T R A C T
Carbon sequestration in tropical soils has potential for mitigating global warming and increasing agricultural productivity. We analyzed 26 long-term experiments (LTEs) in different agro-climatic zones (ACZs) of India to assess the potential and cost of $C$ sequestration. Data on initial and final soil organic C (SOC) concentration in the recommended $\mathrm{N}, \mathrm{P}$ and $\mathrm{K}(\mathrm{NPK})$; recommended $\mathrm{N}, \mathrm{P}$ and $\mathrm{K}$ plus farmyard manure (NPK + FYM) and unfertilized (control) treatments were used to calculate carbon sequestration potential (CSP) i.e., capacity to sequester atmospheric carbon dioxide $\left(\mathrm{CO}_{2}\right)$ by increasing SOC stock, under different nutrient management scenarios. In most of the LTEs wheat equivalent yields were higher in the NPK + FYM treatment than the NPK treatment. However, partial factor productivity (PFP) was more with the NPK treatment. Average SOC concentration of the control treatment was $0.54 \%$, which increased to $0.65 \%$ in the NPK treatment and $0.82 \%$ in the NPK + FYM treatment. Compared to the control treatment the NPK + FYM treatment sequestered $0.33 \mathrm{MgCha}^{-1} \mathrm{yr}^{-1}$ whereas the NPK treatment sequestered $0.16 \mathrm{MgCha}^{-1} \mathrm{yr}^{-1}$. The CSP in different nutrient management scenarios ranged from 2.1 to $4.8 \mathrm{Mg} \mathrm{Cha}$ during the study period (average $16.9 \mathrm{yr}$ ) of the LTEs. In 17 out of 26 LTEs, the NPK + FYM treatment had higher SOC and also higher net return than that of the NPK treatment. In the remaining 9 LTEs SOC sequestration in the NPK + FYM treatment was accomplished with decreased net return suggesting that these are economically not attractive and farmers have to incur into additional cost to achieve $C$ sequestration. The feasibility of SOC sequestration in terms of availability of FYM and other organic sources has been discussed in the paper.
\end{abstract}

(C) 2010 Elsevier B.V. All rights reserved.

\section{Introduction}

Soil is one of the important sources and sinks of greenhouse gases (GHGs) causing global warming and climate change (Janseens et al., 2003). It contributes about 20\% to the total emission of carbon dioxide through soil respiration and root respiration, $12 \%$ of methane and $60 \%$ of anthropogenic nitrous oxide emissions (IPCC, 2007). Global warming may affect global carbon cycle thereby distorting structure and functions of ecosystems. Organic matter concentration, which is quite low $(<1.0 \%)$ in the tropical soils, would become still lower and climatic change may affect its quality (Lal, 2004; Smith et al., 2008). Soil biology and microbial populations are

Abbreviations: ACZ, agro-climatic zone; CDM, clean development mechanism; CSP, carbon sequestration potential; FYM, farmyard manure; GHG, greenhouse gas; GWP, global warming potential; IGP, Indo-Gangetic plain; LTEs, long-term experiments; Mha, million hectares; MSOC, mass of soil organic carbon; Mt, million tons; NPK, nitrogen, phosphorus and potassium; PPF, partial factor productivity; SOC, soil organic carbon; WEY, wheat equivalent yield.

* Corresponding author. Tel.: +91 1125842986.

E-mail address: hpathak.iari@gmail.com (H. Pathak). expected to change under changed climatic conditions (Baker, 2004).

Mitigation of $\mathrm{CO}_{2}$ emission from agriculture can be achieved by increasing $C$ sequestration in soil, which implies storage of $C$ as soil organic matter (Lal, 2004). Judicious nutrient management is crucial to soil organic $\mathrm{C}$ (SOC) sequestration in tropical soils (Bhattacharyya et al., 2007; Mandal et al., 2007). Adequate supply of nutrients in soil can enhance biomass production and SOC content (Van Kessel and Hartley, 2000). Use of organic manure and compost enhances the SOC pool more than application of the same amount of nutrients as inorganic fertilizers (Gregorich et al., 2001). Long-term manure application increases the SOC pool (Gilley and Risse, 2000), which not only sequester $\mathrm{CO}_{2}$ but also enhances productivity of soil (Swarup et al., 2000; Manna et al., 2005). It is, however, argued that SOC sequestration is a major challenge in soils of the tropics and sub-tropics, where climate is harsh and resource-poor farmers cannot afford the input of organic manure and crop residues. The rate of $C$ mineralization is high in the tropics because of high temperature and the humification efficiency is low (Ladha et al., 2003).

Long-term experiments (LTEs) provide opportunities for assessing long-term changes in SOC and crop yields and estimating $\mathrm{C}$ 


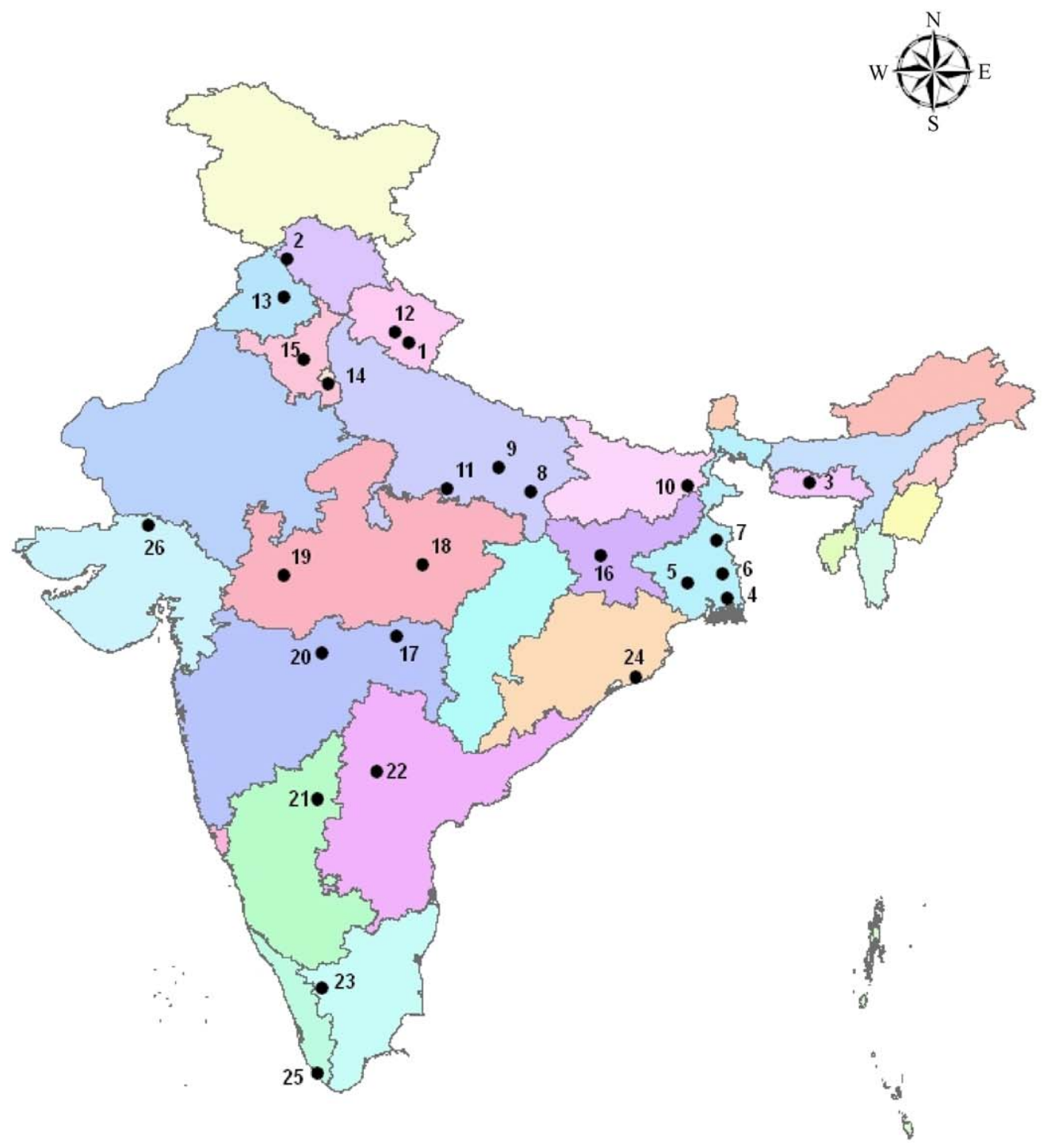

\section{Legend}

1. Ahmora, Uttarakhand

2. Palamp ur, Him achal Pradesh

3. Umiam, Meghalaya

4. Barackp ore, West Bengal

5. Mohanpur, West Bengal

6. Gayeshp ur, West Bengal

7. Kalyani, West Bengal

8. Varanasi, Uttar Pradesh
10. Samastip ur, Bihar

11. Karp ur, Uttar Prad esh

12. Pantnagar, U ttarakhand

13. Ludhiana, Punjab

14. New Delli, Delhi

15. Kamal, Haryana

16. Ranchi, Jharkhand

17. Nagp ur, Maharashtra

18. Jabalp ur, Mad hya Prad esh
19. Indore, Madhya Pradesh

20. Akola, Maharashtra

21. Bellary, Karmataka

22. Hyderab ad, Andhra Prad esh

23. Coimbatore, Tamil Nadu

24. Cuttack, Orissa

25. Trivandrum, Kerala

26. S.K. Nagar, G ujarat

9. Fairahad, U ttar Pradesh

Fig. 1. Location of the long-term experiments in various states of India.

sequestration potential (CSP) of agricultural lands (Powlson et al., 1986; Ladha et al., 2003). They also provide data for calculation of cost of $C$ sequestration on which to base rational judgments about the feasibility of $C$ sequestration (Lal, 2005). Many LTEs began in the 1970s and 1980s in different agro-climatic zones (ACZs) following different cropping systems in India. Using the data from these LTEs, estimates of C sequestration were carried out by some researchers (Swarup et al., 2000; Lal, 2004; Manna et al., 2005; Mandal et al., 2007, 2008). These estimates used older data, fol- lowed different procedures and used only a few LTEs to calculate C sequestration potential. In this paper a combined analysis of 26 LTEs from diverse ACZs in India was made to calculate potential and cost of $C$ sequestration using the most recent data and identical statistical procedure. The objectives of the paper were to (1) monitor change in SOC in 26 LTEs conducted at different ACZs of India, (2) evaluate potential of SOC sequestration in different LTEs and (3) estimate cost of SOC sequestration in agricultural soils of India. 
Table 1

Selected long-term experiments in various agro-climatic zones of India.

\begin{tabular}{|c|c|c|c|c|c|c|}
\hline LTE $^{\mathrm{a}}$ no. & Location & State & Cropping system & Years & Duration (yr) & Reference \\
\hline 1 & Almora & Uttarakhand & Soybean-Wheat & $1973-03$ & 30 & Kundu et al. (2007) \\
\hline 2 & Palampur & Himachal Pradesh & Maize-Wheat & $1972-96$ & 25 & Sharma et al. (1998) \\
\hline 3 & Umiam & Meghalaya & Rice-Wheat & $2000-08$ & 8 & Ghosh et al. (in press) \\
\hline 4 & Barackpore & West Bengal & Rice-Wheat-Jute & $1972-01$ & 28 & Manna et al. (2005) \\
\hline 5 & Mohanpur & West Bengal & Rice-Wheat & 1986-99 & 20 & Mandal et al. (2007) \\
\hline 6 & Gayeshpur & West Bengal & Rice-Mustard-Sesame & 1986-99 & 13 & Mandal et al. (2007) \\
\hline 7 & Kalyani & West Bengal & Rice-Berseem & $1985-05$ & 20 & Majumder et al. (2008) \\
\hline 8 & Varanasi & Uttar Pradesh & Rice-Wheat & $1985-97$ & 12 & Yadav et al. (2000) \\
\hline 9 & Faizabad & Uttar Pradesh & Rice-Wheat & $1984-85$ & 14 & Yadav et al. (2000) \\
\hline 10 & Samastipur & Bihar & Rice-Wheat & $1988-96$ & 8 & Prasad and Sinha (2000) \\
\hline 11 & Kanpur & Uttar Pradesh & Rice-Wheat & 1984-97 & 14 & Yadav et al. (2000) \\
\hline 12 & Pantnagar & Uttarakhand & Rice-Wheat & $1983-97$ & 14 & Yadav et al. (2000) \\
\hline 13 & Ludhiana & Punjab & Rice-Wheat & $1983-97$ & 15 & Yadav et al. (2000) \\
\hline 14 & New Delhi & Delhi & Maize-Wheat-Cowpea (F) & $1971-03$ & 32 & Rudrappa et al. (2006) \\
\hline 15 & Karnal & Haryana & Rice-Wheat & 1994-04 & 10 & Yaduvanshi and Swarup (2005) \\
\hline 16 & Ranchi & Jharkhand & Soybean-Wheat & $1971-02$ & 30 & Manna et al. (2005) \\
\hline 17 & Nagpur & Maharashtra & Cotton-Sorghum & 1986-95 & 9 & Venugopalan and Pundarikakshud (1998) \\
\hline 18 & Jabalpur & Madhya Pradesh & Soybean-Wheat-Maize (F) & $1972-94$ & 28 & Hati et al. (2007) \\
\hline 19 & Indore & Madhya Pradesh & Soybean-Wheat & $1995-00$ & 6 & Behera et al. (2007) \\
\hline 20 & Akola & Maharashtra & Sorghum-Wheat & $1988-01$ & 12 & Manna et al. (2005) \\
\hline 21 & Bellary & Karnataka & Maize-Chickpea & $1978-01$ & 23 & Vineela et al. (2008) \\
\hline 22 & Hyderabad & Andhra Pradesh & Sorghum-Castor & 1995-01 & 6 & Sharma et al. (2005) \\
\hline 23 & Coimbatore & Tamil Nadu & Finger millet-Maize-Cowpea (F) & $1972-92$ & 20 & Murugappan et al. (1998) \\
\hline 24 & Cuttack & Orissa & Rice-Rice & $1984-04$ & 10 & Nayak et al. (2009) \\
\hline 25 & Trivandrum & Kerala & Cassava & 1977-90 & 13 & John et al. (1998) \\
\hline 26 & S.K. Nagar & Gujarat & Pearl millet & $1988-06$ & 18 & Srinivasa Rao et al. (2009) \\
\hline
\end{tabular}

a Long-term experiment.

\section{Materials and methods}

\subsection{Experimental sites}

The mainland of India extends between latitudes $8^{\circ} 4^{\prime} \mathrm{N}$ and $37^{\circ} 6^{\prime} \mathrm{N}$, and longitudes $68^{\circ} 7^{\prime} \mathrm{E}$ and $97^{\circ} 3^{\prime} \mathrm{E}$. With the 329 million hectares (Mha) of the geographical area the country presents a large number of complex agro-climatic conditions. The Planning Commission of India delineated the country into $15 \mathrm{ACZs}$ based on physiography, soils, bio-climatic types and crop growing period to form the basis for agricultural planning.

Data of 26 LTEs conducted with 19 different cropping systems at different ACZs covering 20 states of the country were collected for the present study (Fig. 1). The LTEs were located between the latitudes of $8.2^{\circ} \mathrm{N}$ and $32.0^{\circ} \mathrm{N}$ and longitudes of $79.1^{\circ} \mathrm{E}$ and $92.0^{\circ} \mathrm{E}$ (Table 1). Altitude ranged from $9 \mathrm{~m}$ to $1642 \mathrm{~m}$ above mean sea level. They were situated in sub-tropical to warm temperate climates characterized by cool and dry winters and warm and wet summers. The mean annual maximum and minimum temperatures were $23.0-39.0^{\circ} \mathrm{C}$ and $2.5-23^{\circ} \mathrm{C}$, respectively. Average rainfall ranged from 508 to $2439 \mathrm{~mm}$ and solar radiation ranged from 15.2 to $23.7 \mathrm{MJ} \mathrm{m}^{-2} \mathrm{~d}^{-1}$. The sites varied widely in term of soil type, which included Alfisol, Entisol, Inceptisol, Mollisol, Aridisol and Ultisol. Textures of the experimental soils were sandy loam to clay. Initial SOC in different LTEs was in the range of $0.22 \%$ (LTE 13, Ludhiana, Punjab)-1.58\% (LTE 6, Gayeshpur, West Bengal) (Table 2).

\subsection{Treatments and crop management}

Nineteen out of 26 LTEs included two crops per year i.e., either rice, maize, millets or soybean grown in summer months (June-October) under monsoon climatic conditions and either wheat, mustard or chickpea grown in the cooler and drier winter months (November-March) (Table 1). Five LTEs included three crops whereas only two (LTE 25 and 26) included one crop per year. For the present study, published data from the recommended $\mathrm{N}$, $\mathrm{P}$ and $\mathrm{K}(\mathrm{NPK})$; recommended $\mathrm{N}, \mathrm{P}$ and $\mathrm{K}$ plus farmyard manure $(\mathrm{NPK}+\mathrm{FYM})$ and unfertilized (control) treatments were included.
Addition of N, P, K and FYM; and yield in NPK and NPK + FYM treatments in various long-term experiments are given in Table 3. The LTEs had different crops and cropping systems. Therefore, for comparison of treatments, the yields of different crops were converted into wheat equivalent yield (WEY) as wheat was the most common crop in the LTEs. The following equation was used to calculate WEY:

$\mathrm{WEY}=\frac{\mathrm{GY} 1 \times \mathrm{PY} 1+\mathrm{GY} 2 \times \mathrm{PY} 2+\mathrm{GY} 3 \times \mathrm{PY} 3}{\mathrm{PW}}$

where GY1, GY2 and GY3 are grain (economic) yields ( $\left.\mathrm{Mg} \mathrm{ha}^{-1}\right)$ of crop 1, 2 and 3; PY1, PY2 and PY3 are price of grain for the year 2010 (US\$ $\mathrm{Mg}^{-1}$ ) of crop 1, 2 and 3; respectively and PW is price of wheat $\left(\mathrm{US} \$ \mathrm{Mg}^{-1}\right)$

\subsection{Estimation of $C$ sequestration potential}

The $C$ sequestration was calculated only in terms of increase in $C$ stock in soil. Emission of GHGs such as methane and nitrous oxide were not considered. Data on initial and final SOC concentrations in the NPK, NPK + FYM and unfertilized (control) treatments were collected for all the LTEs. The mass of SOC in the surface layer $(0-15 \mathrm{~cm})$ of soil was calculated as

$\mathrm{MSOC}=\mathrm{SOC} \times \mathrm{BD} \times T$

where MSOC is mass of SOC $\left(\mathrm{Mg} \mathrm{ha}^{-1}\right)$, SOC is organic C concentration in soil (\%), BD is bulk density $\left(\mathrm{Mg} \mathrm{m}^{-3}\right)$ and $T$ is thickness of surface layer $(\mathrm{cm})$. The BD for different treatments was calculated using the following equation (Manrique and Jones, 1991):

$\mathrm{BD}\left(\mathrm{Mg} \mathrm{m}^{-3}\right)=1.51-0.113 \times \mathrm{SOC}(\%)$.

Carbon sequestration potential (CSP) was calculated for three nutrient management scenarios as discussed below.

\subsubsection{Carbon sequestration potential with balanced NPK use (CSP_NPK)}

In this scenario it was assumed that the current low SOC content, which was a result of imbalanced and inadequate use N, P and $\mathrm{K}$ fertilizer in most farmers' fields, could be improved with the use of 
Table 2

Location, soil and climatic characteristics of the long-term experimental sites in India.

\begin{tabular}{|c|c|c|c|c|c|c|c|c|c|c|c|c|c|}
\hline LTE no. & $\begin{array}{l}\text { Latitude } \\
\left({ }^{\circ} \mathrm{N}\right)\end{array}$ & $\begin{array}{l}\text { Longitude } \\
\left({ }^{\circ} \mathrm{E}\right)\end{array}$ & $\begin{array}{l}\text { Altitude } \\
\text { (m) }\end{array}$ & Soil type & $\begin{array}{l}\text { SOC } \\
(\%)\end{array}$ & $\begin{array}{l}\text { Clay } \\
(\%)\end{array}$ & $\begin{array}{l}\text { Silt } \\
(\%)\end{array}$ & $\begin{array}{l}\text { Sand } \\
(\%)\end{array}$ & $\begin{array}{l}\text { Bulk } \\
\text { density } \\
\left(\mathrm{Mg} \mathrm{m}^{-3}\right)\end{array}$ & $\begin{array}{l}\text { Daily solar } \\
\text { radiation } \\
\left(\mathrm{MJ} \mathrm{m}^{-2}\right)\end{array}$ & $\begin{array}{l}\text { Daily } \\
\text { minimum } \\
\text { temp. }\left({ }^{\circ} \mathrm{C}\right)\end{array}$ & $\begin{array}{l}\text { Daily } \\
\text { maximum } \\
\text { temp. }\left({ }^{\circ} \mathrm{C}\right)\end{array}$ & $\begin{array}{l}\text { Annual } \\
\text { rainfall } \\
(\mathrm{mm})\end{array}$ \\
\hline 1 & 29.6 & 79.5 & 1642.0 & Typic Hapluquept & 0.55 & 5.8 & 16.1 & 78.1 & 1.34 & 18.1 & 10.0 & 23.0 & 1079 \\
\hline 2 & 32.0 & 76.0 & 1280.0 & Typic Hapludalf & 0.79 & 15.7 & 67.4 & 16.9 & 1.42 & 17.0 & 13.6 & 23.7 & 2300 \\
\hline 3 & 25.6 & 91.0 & 968.0 & Typic Hapludalf & 1.47 & 32.6 & 27.9 & 39.5 & 1.34 & 16.9 & 2.5 & 32.1 & 2439 \\
\hline 4 & 22.8 & 88.4 & 9.0 & Typic Eutrochrept & 0.71 & 18.0 & 28.0 & 54.0 & 1.35 & 17.9 & 21.2 & 31.3 & 1441 \\
\hline 5 & 23.0 & 89.0 & 10.0 & Typic Endoaquept & 1.42 & 20.8 & 68.0 & 11.2 & 1.12 & 19.0 & 12.5 & 36.2 & 1480 \\
\hline 6 & 23.0 & 89.0 & 10.0 & Typic Haplustept & 1.58 & 22.0 & 63.0 & 15.0 & 1.18 & 18.6 & 12.0 & 36.5 & 1600 \\
\hline 7 & 22.9 & 88.9 & 10.0 & Typic Haplustept & 0.77 & 21.6 & 64.8 & 13.6 & 1.15 & 15.2 & 12.0 & 36.5 & 1470 \\
\hline 8 & 25.3 & 83.0 & 129.0 & Typic Haplustert & 0.42 & 29.0 & 17.0 & 54.0 & 1.46 & 19.2 & 18.0 & 32.3 & 1039 \\
\hline 9 & 26.0 & 82.1 & 113.0 & Udic Fluvents & 0.37 & 12.7 & 63.0 & 24.4 & 1.47 & 17.9 & 18.8 & 30.4 & 1057 \\
\hline 10 & 25.5 & 87.5 & 52.0 & Typic Haplustept & 0.51 & 38.6 & 45.6 & 11.6 & 1.45 & 15.6 & 20.8 & 31.0 & 508 \\
\hline 11 & 25.4 & 80.6 & 129.0 & Aeric Chroqualf & 0.29 & 20.1 & 58.3 & 21.6 & 1.48 & 19.5 & 19.6 & 31.9 & 945 \\
\hline 12 & 29.9 & 79.1 & 244.0 & Udic Ustochrept & 1.4 & 17.8 & 46.0 & 36.0 & 1.49 & 18.3 & 15.7 & 31.1 & 1394 \\
\hline 13 & 30.9 & 75.9 & 247.0 & Typic Ustipsamment & 0.22 & 12.6 & 89.0 & 78.5 & 1.49 & 19.4 & 17.5 & 30.5 & 800 \\
\hline 14 & 28.4 & 77.2 & 250.0 & Typic Haplusept & 0.44 & 14.0 & 16.0 & 69.0 & 1.46 & 20.1 & 6.1 & 39.0 & 750 \\
\hline 15 & 29.1 & 76.5 & 252.0 & Acquic Natrustalf & 0.40 & 24.1 & 24.3 & 51.6 & 1.46 & 19.3 & 17.3 & 31.1 & 580 \\
\hline 16 & 23.5 & 85.0 & 120.0 & Typic Haplustalf & 0.45 & 25.4 & 8.4 & 66.2 & 1.35 & 17.0 & 17.0 & 29.0 & 1545 \\
\hline 17 & 21.2 & 79.1 & 301.0 & Ustochrept & 0.41 & 46.0 & 38.0 & 16.0 & 1.46 & 19.0 & 20.5 & 34.6 & 1050 \\
\hline 18 & 23.2 & 79.9 & 393.0 & Typic Haplustert & 0.32 & 60.4 & 23.0 & 15.9 & 1.47 & 17.9 & 19.2 & 31.3 & 2044 \\
\hline 19 & 22.9 & 75.9 & 520.0 & Typic Haplustert & 0.51 & 52.4 & 16.2 & 31.4 & 1.45 & 18.8 & 20.8 & 34.6 & 848 \\
\hline 20 & 20.7 & 77.0 & 307.0 & Vertisol & 0.46 & 76.0 & 18.0 & 6.0 & 1.26 & 19.2 & 20.5 & 34.3 & 742 \\
\hline 21 & 16.5 & 76.9 & 448.0 & Vertisol & 0.66 & 60.0 & 22.0 & 18.0 & 1.44 & 21.8 & 20.6 & 33.3 & 632 \\
\hline 22 & 17.3 & 78.6 & 220.0 & Typic Kandiustalf & 0.37 & 19.0 & 7.0 & 74.0 & 1.47 & 19.6 & 17.3 & 28.9 & 736 \\
\hline 23 & 11.0 & 76.0 & 379.0 & Typic Hapludoll & 0.57 & 30.0 & 42.0 & 28.0 & 1.45 & 16.9 & 16.6 & 30.6 & 919 \\
\hline 24 & 20.0 & 86.0 & 36.0 & Typic Endoaquept & 0.66 & 20.0 & 14.0 & 66.0 & 1.44 & 16.1 & 21.3 & 31.8 & 1600 \\
\hline 25 & 8.2 & 76.6 & 55.0 & Acid Ultisol & 0.70 & 52.6 & 12.8 & 34.6 & 1.43 & 19.6 & 23.2 & 31.0 & 2352 \\
\hline 26 & 24.5 & 72.7 & 153.0 & Aridisol & 0.43 & 11.7 & 4.1 & 84.1 & 1.5 & 23.7 & 19.2 & 32.2 & 550 \\
\hline
\end{tabular}


Table 3

Addition of N, P, K and FYM; and yield in NPK and NPK + FYM treatments in various long-term experiments.

\begin{tabular}{|c|c|c|c|c|c|c|c|c|c|}
\hline \multirow[t]{2}{*}{ LTE no. } & \multicolumn{4}{|l|}{ NPK treatment } & \multicolumn{5}{|c|}{ NPK + FYM treatment } \\
\hline & $\begin{array}{l}\text { N-P-K crop } 1 \\
\left(\mathrm{~kg} \mathrm{ha}^{-1}\right)\end{array}$ & $\begin{array}{l}\text { N-P-K crop } 2 \\
\left(\mathrm{~kg} \mathrm{ha}^{-1}\right)\end{array}$ & $\begin{array}{l}\text { Crop } 1 \text { yield } \\
\left(\mathrm{Mg} \mathrm{ha}^{-1}\right)\end{array}$ & $\begin{array}{l}\text { Crop } 2 \text { yield } \\
\left(\mathrm{Mg} \mathrm{ha}^{-1}\right)\end{array}$ & $\begin{array}{l}\text { N-P-K crop } 1 \\
\left(\mathrm{~kg} \mathrm{ha}^{-1}\right)\end{array}$ & $\begin{array}{l}\text { N-P-K crop } 2 \\
\left(\mathrm{~kg} \mathrm{ha}^{-1}\right)\end{array}$ & FYM $\left(\mathrm{Mg} \mathrm{ha}^{-1}\right)$ & $\begin{array}{l}\text { Crop } 1 \text { yield } \\
\left(\mathrm{Mg} \mathrm{ha}^{-1}\right)\end{array}$ & $\begin{array}{l}\text { Crop } 2 \text { yield } \\
\left(\mathrm{Mg} \mathrm{ha}^{-1}\right)\end{array}$ \\
\hline 1 & $20-35-33$ & - & 1.4 & 1.1 & $20-35-33$ & - & 10 & 2.8 & 1.9 \\
\hline 2 & $120-26-33$ & $90-26-25$ & 3.3 & 2.8 & $120-26-33$ & $90-26-25$ & 10 & 4.7 & 3.5 \\
\hline 3 & $120-26-25$ & $90-16-0$ & 3.0 & 2.3 & $120-26-25$ & $90-16-0$ & 10 & 4.7 & 3.5 \\
\hline 4 & $120-26-50$ & $120-26-50(60-13-50)^{\mathrm{a}}$ & 3.8 & $2.3(1.8)$ & $120-26-50$ & $120-26-50(60-13-50)$ & 10 & 3.9 & $2.3(2.0)$ \\
\hline 5 & $120-26-33$ & $120-26-33$ & 2.0 & 2.5 & $120-26-33$ & $120-26-33$ & 7.5 & 2.5 & 2.8 \\
\hline 6 & $80-40-40$ & $86-64-53(37-36-15)$ & 2.8 & $1.7(0.6)$ & $80-40-40$ & $86-64-53(37-36-15)$ & 7.5 & 3.3 & $2.0(0.9)$ \\
\hline 7 & $60-40-40$ & $25-50-50$ & 2.7 & 3.4 & $60-40-40$ & $25-50-50$ & 10 & 3.2 & 3.7 \\
\hline 8 & $120-26-33$ & $120-26-33$ & 4.6 & 4.0 & $60-13-16.5$ & $120-26-33$ & 5 & 4.3 & 4.2 \\
\hline 9 & $120-26-33$ & $120-26-33$ & 4.5 & 3.5 & $60-13-16.5$ & $120-26-33$ & 7 & 3.4 & 3.6 \\
\hline 10 & $120-26-33$ & $120-26-33$ & 3.1 & 3.6 & $120-26-33$ & $120-26-33$ & 16 & 3.7 & 4.0 \\
\hline 11 & $120-26-33$ & $120-26-33$ & 3.3 & 4.8 & $60-13-16.5$ & $120-26-33$ & 7.3 & 3.0 & 4.86 \\
\hline 12 & $120-26-33$ & $120-26-33$ & 4.2 & 4.7 & $60-13-16.5$ & $120-26-33$ & 6.3 & 4.0 & 4.4 \\
\hline 13 & $120-26-33$ & $120-26-33$ & 6.1 & 4.0 & $60-13-16.5$ & $120-26-33$ & 5 & 4.8 & 3.9 \\
\hline 14 & $120-26-40$ & $(20-40-20)$ & 2.3 & $4.5(0.5)$ & $120-26-40$ & $(20-40-20)$ & 15 & 2.6 & $5.4(0.7)$ \\
\hline 15 & $120-26-42$ & $120-26-42$ & 4.8 & 3.7 & $120-26-42$ & $120-26-42$ & 10 & 5.3 & 4.1 \\
\hline 16 & $25-26-33$ & $80-26-33$ & 1.4 & 2.3 & $25-26-33$ & $80-26-33$ & 10 & 1.7 & 2.2 \\
\hline 17 & $60-13-25$ & $60-13-25$ & 1.4 & 1.0 & $60-13-25$ & $60-13-25$ & 15 & 0.7 & 1.2 \\
\hline 18 & $20-35-16.6$ & $120-35-33.2(80-26-33)$ & 2.2 & $4.3(6.2)$ & $20-35-16.6$ & $120-35-33.2(80-26-33)$ & 15 & 2.1 & $4.3(7.9)$ \\
\hline 19 & $120-26-33$ & - & 4.7 & 1.8 & $120-26-33$ & - & 10 & 5.8 & 2.0 \\
\hline 20 & $100-50-40$ & $120-60-60$ & 3.4 & 1.6 & $100-50-40$ & $120-60-60$ & 10 & 3.5 & 1.7 \\
\hline 21 & $60-30-30$ & $20-60-20$ & 2.2 & 1.6 & $60-30-30$ & $20-60-20$ & 5 & 2.6 & 1.8 \\
\hline 22 & $60-0-0$ & - & 1.3 & 1.0 & $60-0-0$ & - & 2 & 1.4 & 0.9 \\
\hline 23 & $90-45-17.5$ & $135-67-35(25-50-0)$ & 2.1 & $4.3(1.3)$ & $90-45-17.5$ & $135-67-35(25-50-0)$ & 12.5 & 2.3 & $3.4(0.9)$ \\
\hline 24 & $60-40-40$ & $80-40-40$ & 4.5 & 4.3 & $60-40-40$ & $80-40-40$ & 5 & 4.9 & 4.6 \\
\hline 25 & $100-44-83$ & - & 22.3 & - & $100-44-83$ & - & 12.5 & 29 & - \\
\hline 26 & $50-13-25$ & - & 0.78 & - & $25-6.5-12.5$ & - & 4 & 0.82 & - \\
\hline
\end{tabular}

a Values in the parentheses are for crop 3 in the system 
Table 4

Annual cost, return, wheat equivalent yield and partial factor productivity in the NPK and NPK + FYM treatments in various long-term experiments.

\begin{tabular}{|c|c|c|c|c|c|c|c|c|c|c|}
\hline \multirow[t]{2}{*}{ LTE no. } & \multicolumn{5}{|l|}{ NPK treatment } & \multicolumn{5}{|c|}{ NPK + FYM treatment } \\
\hline & WEYa $\left(\mathrm{Mg} \mathrm{ha}^{-1}\right)$ & Cost (US \$) & Return (US \$) & Benefit:cost & $\mathrm{PFP}^{\mathrm{b}}$ & WEY' $\left(\mathrm{Mg} \mathrm{ha}^{-1}\right)$ & Cost (US \$) & Return (US \$) & Benefit:cost & $\mathrm{PFP}$ \\
\hline 1 & 2.9 & 427.7 & 661.7 & 1.5 & 34.3 & 5.4 & 461.7 & 1246.8 & 2.7 & 24.4 \\
\hline 2 & 5.4 & 670.2 & 1234.0 & 1.8 & 16.5 & 7.2 & 704.3 & 1651.1 & 2.3 & 15.5 \\
\hline 3 & 4.6 & 659.6 & 1061.7 & 1.6 & 15.7 & 7.1 & 693.6 & 1634.0 & 2.4 & 16.4 \\
\hline 4 & 9.2 & 914.9 & 2106.4 & 2.3 & 18.7 & 7.8 & 951.1 & 1783.0 & 1.9 & 12.3 \\
\hline 5 & 4.1 & 789.4 & 940.4 & 1.2 & 9.2 & 5.0 & 814.9 & 1157.4 & 1.4 & 9.2 \\
\hline 6 & 6.6 & 931.9 & 1504.3 & 1.6 & 14.3 & 8.1 & 957.4 & 1863.8 & 1.9 & 14.4 \\
\hline 7 & 3.4 & 659.6 & 783.0 & 1.2 & 14.1 & 3.9 & 693.6 & 889.4 & 1.3 & 10.2 \\
\hline 8 & 7.6 & 678.7 & 1738.3 & 2.6 & 20.8 & 7.6 & 695.7 & 1740.4 & 2.5 & 17.5 \\
\hline 9 & 7.1 & 678.7 & 1627.7 & 2.4 & 19.5 & 6.2 & 704.3 & 1434.0 & 2.0 & 13.6 \\
\hline 10 & 6.0 & 678.7 & 1385.1 & 2.0 & 16.6 & 7.0 & 736.2 & 1600.0 & 2.2 & 11.9 \\
\hline 11 & 7.4 & 678.7 & 1706.4 & 2.5 & 20.5 & 7.2 & 704.3 & 1661.7 & 2.4 & 15.6 \\
\hline 12 & 8.1 & 678.7 & 1851.1 & 2.7 & 22.2 & 7.6 & 702.1 & 1736.2 & 2.5 & 16.8 \\
\hline 13 & 6.5 & 544.7 & 1495.7 & 2.8 & 18.4 & 7.6 & 561.7 & 1748.9 & 3.1 & 18.0 \\
\hline 14 & 7.1 & 648.9 & 1636.2 & 2.5 & 19.2 & 7.4 & 700.0 & 1702.1 & 2.4 & 12.8 \\
\hline 15 & 7.4 & 474.5 & 1710.6 & 3.6 & 20.0 & 8.2 & 510.6 & 1891.5 & 3.7 & 16.1 \\
\hline 16 & 4.1 & 553.2 & 940.4 & 1.7 & 18.9 & 4.4 & 587.2 & 1004.3 & 1.7 & 12.3 \\
\hline 17 & 3.4 & 738.3 & 772.3 & 1.0 & 17.6 & 3.4 & 746.8 & 778.7 & 1.0 & 8.5 \\
\hline 18 & 8.1 & 685.1 & 1853.2 & 2.7 & 19.9 & 13.0 & 738.3 & 2995.7 & 4.1 & 21.2 \\
\hline 19 & 7.7 & 425.5 & 1766.0 & 4.2 & 42.3 & 9.1 & 459.6 & 2102.1 & 4.6 & 28.5 \\
\hline 20 & 5.3 & 538.3 & 1227.7 & 2.3 & 13.9 & 5.4 & 574.5 & 1251.1 & 2.2 & 10.4 \\
\hline 21 & 3.1 & 574.5 & 721.3 & 1.3 & 13.8 & 5.7 & 593.6 & 1306.4 & 2.2 & 19.2 \\
\hline 22 & 2.9 & 259.6 & 670.2 & 2.6 & 44.7 & 2.9 & 266.0 & 672.3 & 2.5 & 31.4 \\
\hline 23 & 5.7 & 834.0 & 1306.4 & 1.6 & 10.5 & 5.2 & 872.3 & 1204.3 & 1.4 & 7.3 \\
\hline 24 & 6.9 & 580.9 & 1591.5 & 2.7 & 23.3 & 7.5 & 597.9 & 1723.4 & 2.9 & 20.5 \\
\hline 25 & 16.5 & 423.4 & 3789.4 & 8.9 & 78.6 & 21.5 & 517.0 & 4936.2 & 9.5 & 56.0 \\
\hline 26 & 0.6 & 85.1 & 140.4 & 1.6 & 6.7 & 0.61 & 87.2 & 146.8 & 1.7 & 4.2 \\
\hline Mean & 6.0 & 608.2 & 1393.1 & 2.0 & 22.0 & 7.0 & 639.7 & 1610.1 & 3.0 & 17.0 \\
\hline
\end{tabular}

a Wheat equivalent yield.

b Partial factor productivity.

recommended levels of N, P and K fertilizer. Sequestration potential of $C$ for this scenario was calculated as

CSP_NPK = MSOC_NPK - MSOC_Control

where CSP_NPK is CSP with balanced NPK use $\left(\mathrm{Mg} \mathrm{ha}^{-1}\right)$, MSOC_NPK is final SOC in the NPK treatment $\left(\mathrm{Mg} \mathrm{ha}^{-1}\right)$ and MSOC_Control is final SOC in the control treatment $\left(\mathrm{Mg} \mathrm{ha}^{-1}\right)$.

\subsubsection{Carbon sequestration potential with FYM plus balanced NPK use (CSP_FYM)}

This scenario assumed that SOC can be improved with addition of FYM over $100 \%$ recommended levels of N, P and K fertilizer. The following equation was used to calculate CSP with this scenario:

CSP_FYM = MSOC_NPK + FYM - MSOC_Control

where CSP_FYM is CSP with FYM plus balanced NPK use $\left(\mathrm{Mg} \mathrm{ha}^{-1}\right)$, MSOC_NPK + FYM is final SOC in the NPK + FYM treatment $\left(\mathrm{Mg} \mathrm{ha}^{-1}\right)$ and MSOC_Control is final SOC in the control treatment $\left(\mathrm{Mg} \mathrm{ha}^{-1}\right)$.

\subsubsection{Carbon sequestration potential with integrated use of NPK} fertilizer and FYM (CSP_INM)

The CSP of the NPK + FYM treatment over the NPK treatment was calculated using the following equation:

CSP_INM = MSOC_NPK + FYM - MSOC_NPK

where CSP_INM is CSP with integrated use of NPK and FYM $\left(\mathrm{Mg} \mathrm{ha}^{-1}\right)$, MSOC_NPK + FYM is final SOC in the NPK + FYM treatment $\left(\mathrm{Mg} \mathrm{ha}^{-1}\right)$ and MSOC_NPK is final SOC in the NPK treatment $\left(\mathrm{Mg} \mathrm{ha}^{-1}\right)$.

Rate of $\mathrm{C}$ sequestration (CSP_Rate) in $\mathrm{Mg} \mathrm{ha}^{-1} \mathrm{yr}^{-1}$ was calculated as

CSP_Rate $=\frac{\text { CSP_S }}{D}$

where CSP_S is CSP in a particular scenario $\left(\mathrm{Mg} \mathrm{ha}^{-1}\right)$ and $D$ is the duration of the LTE (yr).
Carbon sequestration efficiency (CSE) was calculated as

$\mathrm{CSE}=\frac{\text { CSP_Rate_FYM(or CSP_Rate_INM })}{\text { C_FYM }}$

where CSP_Rate_FYM is C sequestration rate in the FYM treatment $\left(\mathrm{Mg} \mathrm{ha}^{-1} \mathrm{yr}^{-1}\right)$, CSP_Rate_INM is C sequestration rate in the $\mathrm{NPK}+\mathrm{FYM}$ treatment $\left(\mathrm{Mg} \mathrm{ha}^{-1} \mathrm{yr}^{-1}\right)$ and C.FYM is amount of $\mathrm{C}$ added through FYM (Mg ha-1 $\left.\mathrm{yr}^{-1}\right)$.

Linear regression analyses were done to determine the relationship between $C$ sequestration rate and various soil, climate and yield parameters in different LTEs. The $P$ values on the slopes were used to test whether $C$ sequestration rates were significantly different from $0(P<0.05)$

\subsection{Economic evaluation}

The cost of cultivation was calculated by taking into account prices of various inputs and outputs. Inputs included seed, fertilizers, biocide, the hiring charges of human labor and machines for land preparation, irrigation, fertilizer application, plant protection, harvesting, and threshing, and the time required per ha to complete an individual field operation (Pathak and Wassmann, 2007; Pathak, 2010). The costs of human and machine labor and various inputs (seed, fertilizer, biocide and fuel) are their current prices in various parts of India collected by a market survey. Gross income was derived using the minimum support price offered by the Government of India for various commodities (grain, economic yield). Net income was calculated as the difference between gross income and total costs of inputs. Partial factor productivity (PFP) was calculated using the following equation:

$\mathrm{PFP}=\frac{\text { Price of produce }\left(\mathrm{Rs} \mathrm{ha}^{-1}\right)}{\text { Price of N, P and K through fertilizer and manure }\left(\mathrm{Rs} \mathrm{ha}^{-1}\right)}$ 


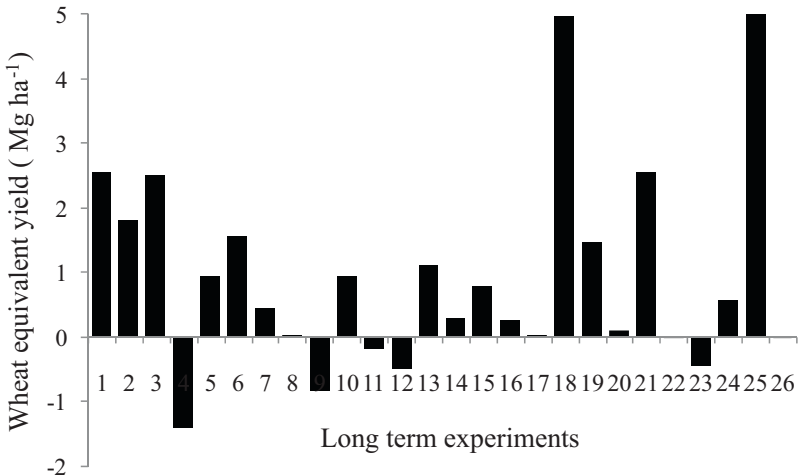

Fig. 2. Change in wheat equivalent yield in the NPK+FYM treatment compared to the NPK treatment in various long-term experiments.

\subsection{Estimation of cost of SOC sequestration}

We considered that use of FYM is an option for $C$ sequestration in tropical soils. This was evident as the NPK + FYM treatments had higher SOC than that of NPK treatment in all the LTEs. These two technologies were compared in terms of annual net returns and annual rate of $\mathrm{C}$ sequestration.

\section{Results and discussion}

\subsection{Yields of crops in the LTES}

Wheat equivalent yield in the NPK treatment varied from $0.6 \mathrm{Mg} \mathrm{ha}^{-1} \mathrm{yr}^{-1}$ in LTE 26 (S.K. Nagar, Gujarat) to $16.5 \mathrm{Mg} \mathrm{ha}^{-1} \mathrm{yr}^{-1}$ in LTE 25 (Trivandrum, Kerala) (Table 4). In the NPK+ FYM treatment the WEY ranged from 0.61 to $21.5 \mathrm{Mg} \mathrm{ha}^{-1} \mathrm{yr}^{-1}$. In most of the LTEs the WEY was higher in the NPK + FYM treatment than the NPK
Long term experiments

$1 \quad 2 \quad 3 \quad 4 \quad 5 \quad 6 \quad 7 \quad 8 \quad 9 \quad 1011121314151617181920212223242526$

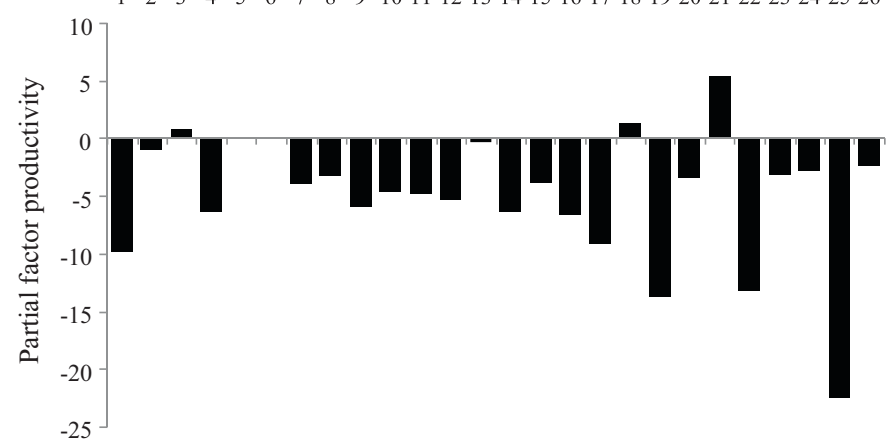

Fig. 3. Changes in partial factor productivity in the NPK + FYM treatment compared to the NPK treatment in various long-term experiments.

treatment (Fig. 2). Improvement in the physico-chemical properties of soil with addition of organic matter as FYM, thereby improving water and nutrient use efficiency were the reasons for higher yield in the NPK + FYM treatment compared to the NPK treatment (Ladha et al., 2003; Hati et al., 2006, 2007). In long-term rice-wheat systems increased yield with addition of organic matter was due to correction of unrecognized nutrient deficiency, indirect effect of nutrient addition such as effect of potassium on resistance to lodging (Swarup et al., 2000; Duxbury, 2001; Manna et al., 2005) and control of soil borne pathogens (Regmi et al., 2002). However, in 5 locations, mostly in the Indo-Gangetic plain (IGP) where intensive rice-wheat cropping system is practiced, the WEY in NPK + FYM was lower to the NPK treatment. Further studies should be carried out to identify the cause of this but higher nutrient supplying capacity of the soils of IGP could be a possible reason (Ladha et al., 2003).

Table 5

Carbon budget and sequestration in the NPK and NPK+FYM treatments in various long-term experiments.

\begin{tabular}{|c|c|c|c|c|c|c|c|c|c|c|c|}
\hline \multirow[t]{2}{*}{ LTE no. } & \multicolumn{3}{|c|}{ Final SOC (\%) } & \multicolumn{3}{|c|}{ C squestration potential $\left(\mathrm{Mg} \mathrm{Cha}^{-1}\right)$} & \multicolumn{3}{|c|}{ Rate of $\mathrm{C}$ sequestration $\left(\mathrm{MgCha} \mathrm{Chr}^{-1}\right)$} & \multicolumn{2}{|c|}{$\begin{array}{l}\text { C sequestration } \\
\text { efficiency }(\%)\end{array}$} \\
\hline & Control & NPK & $\overline{\text { FYM }}$ & CSP_NPK ${ }^{a}$ & CSP_FYM $^{\mathrm{b}}$ & CSP_INM $^{c}$ & CSP_NPK ${ }^{\mathrm{a}}$ & CSP_FYM $^{\mathrm{b}}$ & CSP_INM $^{c}$ & CSP_FYM $^{\mathrm{b}}$ & CSP_INM $^{\mathrm{C}}$ \\
\hline 1 & 0.60 & 0.75 & 1.20 & 3.05 & 11.76 & 8.71 & 0.10 & 0.39 & 0.29 & 11.20 & 8.29 \\
\hline 2 & 0.66 & 0.71 & 1.23 & 1.02 & 11.08 & 10.07 & 0.04 & 0.44 & 0.40 & 12.67 & 11.51 \\
\hline 3 & 1.47 & 1.56 & 1.83 & 1.79 & 4.53 & 2.74 & 0.22 & 0.57 & 0.34 & 16.18 & 9.78 \\
\hline 4 & 0.47 & 0.56 & 0.63 & 1.88 & 3.33 & 1.45 & 0.07 & 0.12 & 0.05 & 3.40 & 1.48 \\
\hline 5 & 0.85 & 1.00 & 1.05 & 2.93 & 3.89 & 0.96 & 0.15 & 0.19 & 0.05 & 7.40 & 1.83 \\
\hline 6 & 0.73 & 1.10 & 1.14 & 7.23 & 8.00 & 0.76 & 0.56 & 0.62 & 0.06 & 23.43 & 2.24 \\
\hline 7 & 0.66 & 0.70 & 0.79 & 0.81 & 2.62 & 1.81 & 0.04 & 0.13 & 0.09 & 3.75 & 2.59 \\
\hline 8 & 0.40 & 0.45 & 0.51 & 0.79 & 2.05 & 1.26 & 0.07 & 0.17 & 0.11 & 9.76 & 6.01 \\
\hline 9 & 0.22 & 0.44 & 0.51 & 4.74 & 6.21 & 1.47 & 0.34 & 0.44 & 0.11 & 18.10 & 4.29 \\
\hline 10 & 0.60 & 0.68 & 0.75 & 1.03 & 2.44 & 1.42 & 0.13 & 0.31 & 0.18 & 5.46 & 3.16 \\
\hline 11 & 0.28 & 0.39 & 0.42 & 2.35 & 3.03 & 0.68 & 0.17 & 0.22 & 0.05 & 8.46 & 1.90 \\
\hline 12 & 0.51 & 0.58 & 0.60 & 1.46 & 1.87 & 0.41 & 0.10 & 0.13 & 0.03 & 6.05 & 1.34 \\
\hline 13 & 0.45 & 0.48 & 0.81 & 0.69 & 7.45 & 6.75 & 0.05 & 0.50 & 0.45 & 28.37 & 25.72 \\
\hline 14 & 1.40 & 1.50 & 2.06 & 1.77 & 11.08 & 9.30 & 0.06 & 0.37 & 0.31 & 7.03 & 5.91 \\
\hline 15 & 0.30 & 0.32 & 0.35 & 0.43 & 1.08 & 0.65 & 0.04 & 0.11 & 0.06 & 3.08 & 1.84 \\
\hline 16 & 0.35 & 0.45 & 0.47 & 1.83 & 2.24 & 0.41 & 0.06 & 0.07 & 0.01 & 2.13 & 0.39 \\
\hline 17 & 0.37 & 0.50 & 0.60 & 2.75 & 4.83 & 2.08 & 0.31 & 0.54 & 0.23 & 10.23 & 4.40 \\
\hline 18 & 0.32 & 0.34 & 0.50 & 0.43 & 3.82 & 3.40 & 0.02 & 0.14 & 0.12 & 2.60 & 2.31 \\
\hline 19 & 0.47 & 0.51 & 0.55 & 0.84 & 1.67 & 0.84 & 0.14 & 0.28 & 0.14 & 7.97 & 3.98 \\
\hline 20 & 0.37 & 0.52 & 0.70 & 3.17 & 6.87 & 3.70 & 0.26 & 0.57 & 0.31 & 16.37 & 8.82 \\
\hline 21 & 0.65 & 0.67 & 0.69 & 0.39 & 0.84 & 0.45 & 0.02 & 0.04 & 0.02 & 2.08 & 1.13 \\
\hline 22 & 0.50 & 0.53 & 0.61 & 0.69 & 2.34 & 1.66 & 0.11 & 0.39 & 0.28 & 55.81 & 39.46 \\
\hline 23 & 0.45 & 0.56 & 0.68 & 2.30 & 4.77 & 2.47 & 0.10 & 0.20 & 0.10 & 4.54 & 2.35 \\
\hline 24 & 0.68 & 0.81 & 0.83 & 2.62 & 3.01 & 0.40 & 0.26 & 0.30 & 0.04 & 17.22 & 2.27 \\
\hline 25 & 0.23 & 0.60 & 0.98 & 7.86 & 15.45 & 7.59 & 0.60 & 1.19 & 0.58 & 27.16 & 13.34 \\
\hline 26 & 0.15 & 0.20 & 0.24 & 1.10 & 1.98 & 0.88 & 0.06 & 0.11 & 0.05 & 7.85 & 3.48 \\
\hline Mean & 0.54 & 0.65 & 0.80 & 2.15 & 4.93 & 2.78 & 0.16 & 0.33 & 0.17 & 12.24 & 6.53 \\
\hline
\end{tabular}

a CSP_NPK, NPK treatment compared to control treatment.

b CSP_FYM, NPK + FYM treatment compared to control treatment.

c CSP_INM, NPK + FYM treatment compared to NPK treatment. 


\subsection{Benefit:cost and partial factor productivity}

The benefit:cost $(\mathrm{B}: \mathrm{C})$ in the major cropping systems across the LTEs varied widely (Table 4). In the NPK treatment B:C values ranged from 0.6 (LTE 26, S.K. Nagar, Gujarat) to 8.9 (LTE 25, Trivandrum, Kerala). In the NPK+ FYM treatment it ranged from 1.0 to 9.5 . In the LTEs the average B:C was 2.0 in the NPK treatment and 3.0 in the NPK + FYM treatment. Both the treatments thus seemed to be profitable as $\mathrm{B}: \mathrm{C}$ was more than 1.0.

The PFP ranged from 6.7 to 78.6 in the NPK treatment and 4.2 to 56.0 in the NPK+FYM treatment (Table 4). In majority of the LTEs, PFP in the NPK treatment was more than the NPK + FYM treatment (Fig. 3). This suggested that yield increase due to addition of nutrient through FYM is lower than that through fertilizer. The relative change in PFP in the NPK + FYM treatment compared to the NPK treatment was highest in LTE 21 (Bellary, Karnataka) followed by LTE 18 (Jabalpur, Madhya Pradesh) and LTE 3 (Umiam, Meghalaya) and was lowest in LTE 25 (Trivandrum, Kerala). Low PFP in the NPK + FYM treatment might be due to slow mineralization of nutrients (Manna et al., 2005). The LTEs with higher relative change in PFP had low sand content $(<15 \%)$ and relative lower SOC concentration. Coarse textured soils in the sub-tropics possess high $\mathrm{C}$ turnover rates because of favorable soil moisture and temperature conditions (Singh et al., 2004).

\subsection{Carbon sequestration}

The final SOC concentrations in both NPK and NPK + FYM treatments were higher than the control treatment (Table 5). Compared to the NPK treatment also, the NPK + FYM treatment had higher SOC concentration in all the LTEs (Fig. 4). The highest increase in SOC in the NPK + FYM treatment was observed in LTE 14 in New Delhi. Organic sources of nutrient such as FYM decompose slowly resulting in more SOC accumulation in soil (Mandal et al., 2007). High lignin content in FYM resulted in greater accumulation of C compared to other non-lignin materials (Paustian et al., 1992).

Carbon sequestration potential (CSP) i.e., increase in soil C stock in a treatment compared to reference treatment in different scenarios varied in the order of CSP_FYM $>$ CSP_INM $>$ CSP_NPK (Table 5). In the CSP_FYM scenario average CSP was $4.93 \mathrm{Mg} \mathrm{Cha}^{-1}$ followed by CSP_INM and CSP_NPK scenarios with CSP of $2.78 \mathrm{Mg} \mathrm{Cha}^{-1}$ and $2.15 \mathrm{MgCha}^{-1}$, respectively. Carbon sequestration in CSP_NPK scenario denoted that even without any organic matter application soils could sequester organic carbon through balanced application of NPK. But application of FYM along with inorganic fertilizer led to an additional build up of SOC in soil. Average rate of

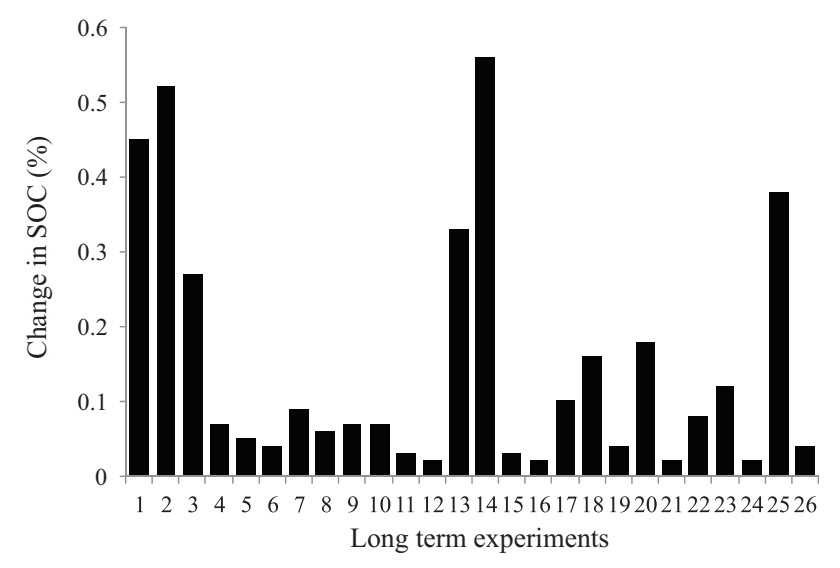

Fig. 4. Change in soil organic $C$ in the NPK + FYM treatment compared to the NPK treatment in various long-term experiments.

sequestration was $0.33 \mathrm{MgCha}^{-1} \mathrm{yr}^{-1}$ in the NPK + FYM treatment whereas in the NPK treatment the rate was $0.16 \mathrm{MgCha}^{-1} \mathrm{yr}^{-1}$. The $C$ sequestration rate was lowest in LTE 18 in the NPK treatment $\left(0.02 \mathrm{MgCha}^{-1} \mathrm{yr}^{-1}\right)$ whereas it was highest in LTE 25 $\left(1.2 \mathrm{MgCha}^{-1} \mathrm{yr}^{-1}\right)$ in the NPK + FYM treatment. In the INM scenario i.e., NPK + FYM treatment compared to the NPK treatment, the average $C$ sequestration rate was $0.17 \%$. Lal (2004) summarized the results of a number of studies and concluded that improved fertility management can enhance the SOC content at the rate of $0.05-0.15 \mathrm{Mg} \mathrm{ha}^{-1} \mathrm{yr}^{-1}$. Manna et al. (2005) observed that application of fertilizer NPK, either alone or in combination with FYM maintained active and slow-release pools of $C$, sequestered $C$ and improved soil quality and productivity.

Soil C sequestration efficiency in the CSP_FYM scenario was maximum (55.81\%) in LTE 22 (Hyderabad, Andhra Pradesh) and minimum $2.08 \%$ in LTE 21 at Bellary, Karnataka. Such large variation could be due to differences in climate, soil and agronomic management parameters. Quality and quantity of FYM also varied across the locations. An average sequestration efficiency of $12.24 \%$ was calculated across the LTEs (Table 5). In the CSP_INM scenario i.e., NPK + FYM treatment compared to the NPK treatment, the average $\mathrm{C}$ sequestration efficiency was $6.53 \%$.

Relationship between various soil, climatic and yield parameters with soil $C$ sequestration rate showed that only yield $(P=0.02)$, latitude $(P=0.03)$ and duration of the experiment $(P=0.03)$ were significantly correlated with $C$ sequestration in the NPK treatment (Table 6). In the NPK + FYM treatment, however, only yield was

Table 6

Relationship between soil, climatic and yield parameters with soil C sequestration.

\begin{tabular}{|c|c|c|c|c|}
\hline \multirow[t]{2}{*}{ Bio-physical parameter } & \multicolumn{2}{|c|}{ Rate of C seq. in the NPK treatment } & \multicolumn{2}{|c|}{ Rate of C seq. in the NPK + FYM treatment } \\
\hline & $R^{2}$ & $P$ value & $R^{2}$ & $P$ value \\
\hline Soil organic C & 0.11 & 0.10 & 0.02 & 0.52 \\
\hline Clay content & 0.04 & 0.33 & 0.04 & 0.31 \\
\hline Silt content & 0.00 & 0.77 & 0.00 & 0.79 \\
\hline Sand content & 0.08 & 0.16 & 0.01 & 0.60 \\
\hline Bulk density & 0.05 & 0.28 & 0.00 & 0.77 \\
\hline Solar radiation & 0.00 & 0.92 & 0.00 & 0.99 \\
\hline Minimum temp. & 0.02 & 0.55 & 0.00 & 0.90 \\
\hline Maximum temp. & 0.04 & 0.32 & 0.00 & 0.88 \\
\hline Average temp. & 0.05 & 0.27 & 0.00 & 0.84 \\
\hline Rainfall & 0.11 & 0.09 & 0.15 & 0.05 \\
\hline Latitude & 0.18 & $0.03^{*}$ & 0.09 & 0.14 \\
\hline Longitude & 0.03 & 0.36 & 0.01 & 0.72 \\
\hline Altitude & 0.05 & 0.27 & 0.01 & 0.61 \\
\hline Duration & 0.17 & $0.03^{*}$ & 0.10 & 0.12 \\
\hline Yield & 0.21 & $0.02^{*}$ & 0.31 & $0.01^{*}$ \\
\hline
\end{tabular}

Significant at $5 \%$ level. 


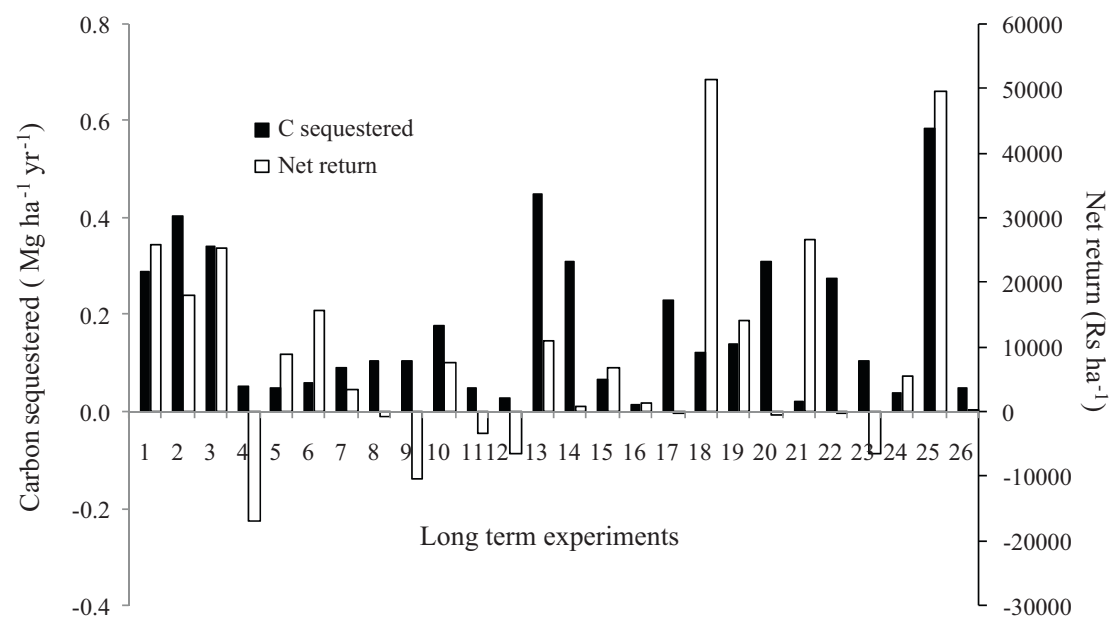

Fig. 5. Sequestration of $C$ with change in net return in the NPK+ FYM treatment compared to the NPK treatment in various long-term experiments.

positively related $(P=0.01)$ with $C$ sequestration. Increased yield results in addition of $C$ through larger amount of root and crop residues and eventual build up of $C$ in the soil because of overall increased biomass production (Mandal et al., 2007). On a long-term basis, increased crop yield and organic matter returned to the soil with manure application results in higher SOC content and biological activity than under controlled condition. Majumder et al. (2008) observed that balanced fertilization caused a net enrichment of both the total carbon and organic carbon content of the soils in rice-wheat-jute rotation because of a large amount of crop residues and root biomass $\mathrm{C}$ left over in the soil owing to the significantly higher yield of the crops grown under those treatments compared to the control. They also observed a significant positive linear relationship between the changes in SOC and the total cumulative crop residue $C$ inputs to the soils over the years. However, Manna et al. (2005) reported that yield of rice and wheat decreased in some of the LTEs though SOC content increased. Rainfall also had good correlation in both the treatments but only at $P=0.09$ and $P=0.05$ levels of significance in the NPK and NPK + FYM treatment (Table 6). Organic matter content across soils is influenced strongly by rainfall. Lal (2004) reported that SOC concentration increased with increase rainfall in several Indian soils.

\subsection{Cost of SOC sequestration}

In 17 of 26 LTEs, the NPK + FYM treatment had higher SOC and also higher net return than that of the NPK treatment (Fig. 5). Application of FYM with NPK thus seemed to be a cost-effective, win-win technology in these LTEs. Because of this win-win nature, the practice of FYM application is widely followed by the farmers, wherever it is feasible. However, in the remaining 9 LTEs SOC sequestration in the NPK + FYM treatment was accomplished with decreased net return (Fig. 5) suggesting that these are economically not attractive and farmers have to incur into additional cost to achieve $C$ sequestration. Therefore, to include farmland as a potential option for $\mathrm{C}$ sequestration, additional financial support through incentives or transferable $\mathrm{C}$ credits under clean development mechanism (CDM) is necessary.

\subsection{Feasibility of SOC sequestration}

With a net cultivated area of 147.43 Mha and current cropping intensity of $135 \%$, the total gross cropped area in the country is 190 Mha (FAI, 2007). The country has a net irrigated area of 54.68 Mha and a gross irrigated area of 75.14 Mha. Among the crops, rice occupies the largest area (44.9 Mha) with a production of 134.0 million tons (Mt) followed by wheat with an area of 27.4 Mha and a production of $75.6 \mathrm{Mt}$.

The study showed that $C$ sequestration in Indian agricultural soil was feasible with application of NPK and FYM. Addition of about $10 \mathrm{Mg}$ FYM ha ${ }^{-1}$ sequestered $0.33 \mathrm{MgCha}^{-1} \mathrm{yr}^{-1}$. However, to apply this magnitude of FYM to 147.43 Mha agricultural land (Table 1$)$, the country would require $1474 \mathrm{Mt}$ of FYM per year ( 737 Mt dry weight considering $50 \%$ moisture content). India supports the largest bovine (cattle + buffalo) population (286.22 million) of the world (MAC, 2006). In the country $335 \mathrm{Mt}$ dung is produced per annum out of which $110 \mathrm{Mt}$ is lost during collection or used for construction purposes (Pathak et al., 2009). The remaining $225 \mathrm{Mt}$ dung is available for use in agriculture. This is only about $1 / 3$ rd of the FYM requirement of the country to achieve the full potential of $C$ sequestration. There are, however, some other sources of organic $C$, which can be used in agriculture for $C$ sequestration. For example, total municipal solid waste (MSW) generation from major Indian cities (35 metro cities and 24 state capitals) is about 40 thousand $\mathrm{Mg} \mathrm{d}^{-1}$ (СРCB, 2006). The biodegradable organic matter in MSW is about $28 \%$ by mass. Thus MSW from Indian cities

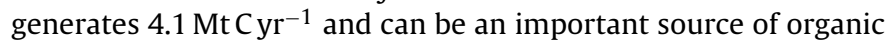
C (Pathak et al., 2009). India also produces about $500 \mathrm{Mt}$ of crop residues annually (Reddy et al., 2002), which can also used in agriculture. However, feasibility of using these materials and their $\mathrm{C}$ sequestration potential requires further study.

\section{Conclusions}

The current study showed that the NPK + FYM treatment have good potential in $C$ sequestration in Indian soils and mitigating GHG emission without any additional cost. Rather it increased yield and net return in majority of the experiments. Increasing SOC in soil makes soil more productive leading to increased crop yield. Thus FYM application was a 'win-win' technology increasing farm income and also sequestering $\mathrm{C}$. In some of the locations, however, application of FYM for $C$ sequestration involved additional expenditure and reduced the net income of the farmers. The technologies of SOC sequestration, therefore, need to be promoted by providing incentives, technological know-how, required resources and policy support to the farmers.

\section{Acknowledgements}

The authors acknowledge the help received from Drs. B. Mandal, P. Ghosh, M. Manna, N. Jain, A. Bhatia from Indian Council of Agri- 
cultural Research, New Delhi for sharing information and providing comments and suggestions on the manuscript.

\section{References}

Baker, J.M., 2004. Yield responses of southern US rice cultivars to $\mathrm{CO}_{2}$ and temperature. Agric. Forest Meteorol. 122, 129-137.

Behera, U., Sharma, A., Pandey, H., 2007. Sustaining productivity of wheat-soybean cropping system through integrated nutrient management practices on the Vertisols of central India. Plant Soil 297, 185-199.

Bhattacharyya, T., Pal, D.K., Easter, M., et al., 2007. Modeled soil organic carbon stocks and changes in the Indo-Gangetic Plains, India from 1980 to 2030. Agric. Ecosyst. Environ. 122, 84-94.

CPCB, 2006. Waste Generation and Composition Report. Central Pollution Control Board, Delhi.

Duxbury, J.M., 2001. Long term yield trends in the rice-wheat cropping system: Results from experiments in north west India. J. Crop Prod. 3, 27-52.

FAI, 2007. Fertilizer Statistics. Fertilizer Association of India, New Delhi.

Ghosh, P.K., Das, A., Saha, R., Khakrang, Tripathi, A.K., Munda, G.C., Ngachan, S.V., in press. Long-term effect on soil quality in acid soil of North-East India. Curr. Sci.

Gilley, J.E., Risse, L.M., 2000. Runoff and soil loss as affected by the application of manure. Trans. Am. Soc. Agric. Biol. Eng. 43, 1583-1588.

Gregorich, E.G., Drury, C.F., Baldock, J.A., 2001. Changes in soil carbon under longterm maize in monoculture and legume-based rotation. Can. J. Soil Sci. 81, 21-31.

Hati, K.K., Swarup, A., Dwivedi, A.K., et al., 2007. Changes in soil physical properties and organic carbon status at the topsoil horizon of a Vertisol of central India after 28 years of continuous cropping, fertilization and manuring. Agric. Ecosyst. Environ. 119, 127-134

Hati, K.K., Mandal, K.G., Misra, A.K., et al., 2006. Effect or inorganic fertilizer and farmyard manure on soil properties, root distribution, and water-use efficiency of soyabean in Vertisols of central India. Bioresour. Technol. 97, 2182-2188.

IPCC, 2007. Climate Change 2007: Climate Change Impacts, Adaptation and Vulnerability. Summary for Policy Makers. Inter-Governmental Panel on Climate Change.

Janseens, I.A., Freibauer, A., Ciais, P., et al., 2003. Europe's biosphere absorbs 7-12\% of anthropogenic carbon emissions. Science 300, 1538-1542.

John, S.K., Mohan Kumar, C.R., Ravindran, C.S., et al., 1998. Long-term effect of manures and fertilizers on cassava production and soil productivity in an acid ultisol. In: Swarup, A., et al. (Eds.), Proceedings of National Workshop on Longterm Soil Fertility Management through Integrated Plant Nutrient Supply. Indian Institute of Soil Science, Bhopal, p. 335

Kundu, S., Bhattacharyya, R., Prakash, V., et al., 2007. Carbon sequestration and relationship between carbon addition and storage under rainfed soybean-wheat rotation in a sandy loam soil of the Indian Himalayas. Soil Tillage Res. 92, 87-95.

Ladha, J.K., Dawe, D., Pathak, H., et al., 2003. How extensive are yield declines in long-term rice-wheat experiments in Asia? Field Crops Res. 81, 159-180.

Lal, R., 2004. Soil carbon sequestration in India. Climatic Change 65, 277-296.

Lal, R., 2005. Soil carbon sequestration for sustaining agricultural production and improving the environment with particular reference to Brazil. J. Sustain. Agric. $26,23-42$.

MAC, 2006. Livestock Census, Department of Animal Husbandry and Dairying, Ministry of Agriculture and Cooperation, Government of India, www.dahd.nic.in.

Majumder, B., Mandal, B., Bandyopadhyay, P., 2008. Soil organic carbon pools and productivity in relation to nutrient management in a 20-year-old rice-berseem agroecosystem. Biol. Fertil. Soils 44, 451-461.

Mandal, B., Majumder, B., Bandyopadhyay, P.K., et al., 2007. The potential of cropping systems and soil amendments for carbon sequestration in soils under long-term experiments in subtropical India. Global Change Biol. 13, 357-369.

Mandal, B., Majumder, B., Adhya, T.K., et al., 2008. Potential of double-cropped rice ecology to conserve organic carbon under subtropical climate. Global Change Biol. 14, 2139-2151.

Manna, M.C., Swarup, A., Wajari, R.H., et al., 2005. Long-term effect of fertilizer and manure application on soil organic carbon storage, soil quality and yield sustainability under sub-humid and semi-arid tropical India. Field Crops Res. 93, 264-280.

Manrique, L.A., Jones, C.A., 1991. Bulk density of soil in relation to soil physical and chemical properties. Soil Sci. Soc. Am. J. 55, 476-481.

Murugappan, V., Santhy, P., Selvi, D., et al., 1998. Long term fertilizer experiment with intensive cropping on an inceptisol. In: Swarup, A., et al. (Eds.), Proceedings of
National Workshop on Long-term Soil Fertility Management through Integrated Plant Nutrient Supply. Indian Institute of Soil Science, Bhopal, p. 335.

Nayak, P., Patel, D., Ramakrishnan, B., et al., 2009. Long-term application effects of chemical fertilizer and compost on soil carbon under intensive rice-rice cultivation. Nutr. Cycl. Agroecosyst. 83, 259-269.

Pathak, H., 2010. Mitigating greenhouse gas and nitrogen loss with improved fertilizer management in rice: quantification and economic assessment. Nutr. Cycl. Agroecosyst. 87, 443-454.

Pathak, H., Jain, N., Bhatia, A., et al., 2009. Global warming mitigation potential of biogas plants in India. Environ. Monit. Assess. 157, 407-418.

Pathak, H., Wassmann, R., 2007. Introducing greenhouse gas mitigation as a development objective in rice-based agriculture. I. Generation of technical coefficients. Agric. Syst. 94, 807-825.

Paustian, K., Parton, W.J., Persson, J., 1992. Modeling soil organic matter in organic amended and nitrogen fertilized long-term plots. Soil Sci. Soc. Am. J. 56, 476-488.

Powlson, D.S., Pruden, G., Johnston, A.E., Jenkinson, D.S., 1986. The nitrogen cycle in Broadbalk wheat experiment - recovery and losses of ${ }^{15} \mathrm{~N}$-labelled fertility applied in springs and inputs of $\mathrm{N}$ from the atmosphere. J. Agric. Sci. (Camb.) 107, 591-609.

Prasad, B., Sinha, S.K., 2000. Long-term effects of fertilizers and organic manures on crop yields, nutrient balance, and soil properties in rice-wheat cropping system in Bihar. In: Abrol, I.P., et al. (Eds.), Long-term Soil Fertility Experiments in Rice-Wheat Cropping Systems. Rice-Wheat Consortium Paper Series, vol. 6. Rice-Wheat Consortium for the Indo-Gangetic Plains, New Delhi, India.

Reddy, K.S., Singh, M., Swarup, A., 2002. Sulfur mineralization in two soils amended with organic manures, crop residues, and green manures. J. Plant Nutr. Soil Sci. 165, 167-171.

Regmi, A.P., Ladha, J.K., Pathak, H., et al., 2002. Analyses of yield and soil fertility trends in a 20-year rice-rice-wheat experiment in Nepal. Soil Sci. Soc. Am. J. 66 857-867.

Rudrappa, L., Purakayastha, T.J., Singh, D., et al., 2006. Long-term manuring and fertilization effects on soil organic carbon pools in a Typic Haplustept of semi-arid sub-tropical India. Soil Tillage Res. 88, 180-192.

Sharma, K.L., Mandal, U.K., Srinivasa, K., et al., 2005. Long-term soil management effects on crop yields and soil quality in a dryland Alfisol. Soil Tillage Res. 83, 246-259.

Sharma, S.P., Sharma, J., Subehia, S.K., 1998. Long-term effects of chemical fertilizers on crop yields, nutrients uptake and soil environment in western himalayan soils. In: Swarup, A., et al. (Eds.), Proceedings of National Workshop on Longterm Soil Fertility Management through Integrated Plant Nutrient Supply. Indian Institute of Soil Science, Bhopal, p. 335.

Singh, J., Blaise, D., Rao, K.V., et al., 2004. On-farm evaluation of integrated nutrient management and moisture-conservation practices on rainfed upland cotton (Gossypium hirsutum). Indian J. Agri. Sci. 74, 649-653.

Smith, P., Fang, C., Dawson, J.J.C., et al., 2008. Impact of global warming on soil organic carbon. Adv. Agron. 97, 1-43.

Srinivasa Rao, Ch., Chary, G.R., Venkateswarlu, B., et al., 2009. Carbon Sequestration Strategies in Rainfed Production Systems of India. Central Research Institute for Dryland Agriculture, ICAR, Hyderabad 500059, India.

Swarup, A., Manna, M.C., Singh, G.B., 2000. Impact of land use and management practices on organic carbon dynamics in soils of India. In: Lal, R., Kimble, J.M., Stewart, B.A. (Eds.), Global Climate Change and Tropical Ecosystems, Advances in Soil Science. CRC Press, Boca Raton, FL, pp. 261-281.

Van Kessel, C., Hartley, C., 2000. Agricultural management of grain legumes: has it led to an increase in nitrogen fixation? Field Crops Res. 65, 165-181.

Venugopalan, M.V., Pundarikakshud, R., 1998. Long-term fertilizer experiment in Cotton based cropping in rainfed Vertisols. In: Swarup, A. (Ed.), Proceedings of National Workshop on Long-term Soil Fertility Management through Integrated Plant Nutrient Supply. Indian Institute of Soil Science, Bhopal, p. 335.

Vineela, C., Wani, S.P., Srinivasarao, C., et al., 2008. Microbial properties of soils as affected by cropping and nutrient management practices in several longterm manural experiments in the semi-arid tropics of India. Appl. Soil Ecol. 40, $165-173$.

Yadav, R.L., Dwivedi, B.S., Prasad, K., et al., 2000. Yield trends, and changes in soil organic-C and available NPK in a long-term rice-wheat system under integrated use of manures and fertilisers. Field Crops Res. 68, 219-246.

Yaduvanshi, N.P.S., Swarup, A., 2005. Effect of continuous use of sodic irrigation water with and without gypsum, farmyard manure, press mud and fertilizer on soil properties and yields of rice and wheat in a long term experiment. Nutr. Cycl. Agroecosyst. 73, 111-118. 